PROCEEDINGS OF THE

AMERICAN MATHEMATICAL SOCIETY

Volume 137, Number 5, May 2009, Pages 1763-1770

S 0002-9939(08)09749-9

Article electronically published on December 11, 2008

\title{
MAPS PRESERVING THE GEOMETRIC MEAN OF POSITIVE OPERATORS
}

\author{
LAJOS MOLNÁR
}

(Communicated by Marius Junge)

\begin{abstract}
Let $H$ be a complex Hilbert space. The symbol $A \# B$ stands for the geometric mean of the positive bounded linear operators $A, B$ on $H$ in the sense of Ando. In this paper we describe the general form of all automorphisms of the set of positive operators with respect to the operation \#. We prove that if $\operatorname{dim} H \geq 2$, any such transformation is implemented by an invertible bounded linear or conjugate-linear operator on $H$.
\end{abstract}

\section{InTRoduction AND STATEMENT OF THE MAIN RESUlt}

Let $H$ be a complex Hilbert space with inner product $\langle.,$.$\rangle . Denote by B(H)$ the algebra of all bounded linear operators on $H$. An operator $A \in B(H)$ is called positive if $\langle A x, x\rangle \geq 0$ holds for every $x \in H$ in which case we write $A \geq 0$. The set of all positive operators on $H$ is denoted by $B(H)^{+}$.

In [1] Ando defined the geometric mean of any two positive operators $A, B \in$ $B(H)^{+}$by the formula

$$
A \# B=\max \left\{X \geq 0:\left[\begin{array}{ll}
A & X \\
X & B
\end{array}\right] \geq 0\right\}
$$

and studied its various properties. This concept is well known to play an important role in matrix theory and operator theory, but recently serious applications have been given also in certain parts of quantum information theory (see, e.g., [2, pp. 247 and 336]).

Apparently, \# is a binary operation on the set of all positive operators. The aim of this paper is to determine the corresponding automorphisms of the groupoid $B(H)^{+}$. Before presenting the main result we observe the following. It is clear that \# has a certain connection with the usual operator product. In the paper [7 Šemrl described the semigroup isomorphisms between so-called standard operator algebras with respect to that product. It might be interesting to observe the differences between the structures of these two kinds of isomorphisms.

Received by the editors November 13, 2007, and, in revised form, June 27, 2008.

2000 Mathematics Subject Classification. Primary 47B49, 47A64.

Key words and phrases. Geometric mean, positive operators, automorphism.

The author was supported by the Hungarian National Foundation for Scientific Research (OTKA), Grant No. T046203, NK68040 and by the Alexander von Humboldt Foundation, Germany.

(C)2008 American Mathematical Society Reverts to public domain 28 years from publication 
Theorem. Let $H$ be a complex Hilbert space with $\operatorname{dim} H \geq 2$. Suppose that $\phi$ : $B(H)^{+} \rightarrow B(H)^{+}$is a bijective map which satisfies

$$
\phi(A \# B)=\phi(A) \# \phi(B) \quad\left(A, B \in B(H)^{+}\right) .
$$

Then there is an invertible bounded linear or conjugate-linear operator $S$ on $H$ such that $\phi$ is of the form

$$
\phi(A)=S A S^{*} \quad\left(A \in B(H)^{+}\right) .
$$

It follows from our result that the automorphism $\phi$ above is automatically additive and positive homogeneous on the cone of all positive operators.

Remark. In the theorem it is assumed that $\operatorname{dim} H \geq 2$. What concerns the onedimensional case, the problem clearly reduces to the question of determining all bijective functions on the set $\mathbb{R}_{+}$of all nonnegative real numbers which respect the operation of the usual geometric mean. It is easy to see that those functions are exactly the positive scalar multiples of the multiplicative bijections of $\mathbb{R}_{+}$. The form of any bijective multiplicative function $f: \mathbb{R}_{+} \rightarrow \mathbb{R}_{+}$can easily be determined as follows. Obviously, $f$ maps positive numbers to positive numbers. Therefore, the function $t \mapsto \log f\left(e^{t}\right)$ is a well-defined additive bijection on the set of all real numbers. Denote it by $a$. We obtain that the general form of $f$ is

$$
f(t)= \begin{cases}0, & \text { if } t=0 \\ e^{a(\log t)}, & \text { if } t>0\end{cases}
$$

where $a$ is any bijective additive function on $\mathbb{R}$. This shows that in the onedimensional case the studied transformations can be very irregular.

\section{ProOF}

First we list some of the basic properties of the geometric mean that we shall use in the proof. For arbitrary selfadjoint operators $A, B \in B(H)$ we write $A \leq B$ if and only if $B-A \geq 0$. All the operators appearing in the following list of properties are supposed to belong to $B(H)^{+}$with the exception of $S$ appearing in (ii).

(i) If $A \leq C$ and $B \leq D$, then $A \# B \leq C \# D$.

(ii) (Transfer property) We have $S(A \# B) S^{*}=\left(S A S^{*}\right) \#\left(S B S^{*}\right)$ for every invertible bounded linear or conjugate-linear operator $S$ on $H$.

(iii) Suppose $A_{1} \geq A_{2} \geq \ldots \geq 0, B_{1} \geq B_{2} \geq \ldots \geq 0$ and $A_{n} \rightarrow A, B_{n} \rightarrow B$ strongly. Then we have that $A_{n} \# B_{n} \rightarrow A \# B$ strongly.

(iv) $A \# B=B \# A$.

(v) $A \# B=A^{1 / 2}\left(A^{-1 / 2} B A^{-1 / 2}\right)^{1 / 2} A^{1 / 2}$ if $A$ is invertible.

The transfer property shows that the transformations $A \longmapsto S A S^{*}$ for arbitrary invertible bounded linear or conjugate-linear operators $S$ are all bijective maps of $B(H)^{+}$respecting the operation of the geometric mean. The content of our theorem is that the converse is also true: there is no other kind of transformation having this property.

In the proof we shall need the following lemma in which we use the concept called the strength of a positive operator along a ray. This notion was originally introduced by Busch and Gudder in [3] for the so-called Hilbert space effects (i.e., positive operators bounded by the identity) in the place of positive operators. Let 
$A$ be a positive operator on $H$, consider a unit vector $\varphi$ in $H$ and denote by $P_{\varphi}$ the rank-one projection onto the subspace generated by $\varphi$. The quantity

$$
\lambda\left(A, P_{\varphi}\right)=\sup \left\{\lambda \in \mathbb{R}_{+}: \lambda P_{\varphi} \leq A\right\}
$$

is called the strength of $A$ along the ray represented by $\varphi$. According to 3 , Theorem 4] we have the following formula for the strength:

$$
\lambda\left(A, P_{\varphi}\right)= \begin{cases}\left\|A^{-1 / 2} \varphi\right\|^{-2}, & \text { if } \varphi \in \operatorname{rng}\left(A^{1 / 2}\right) ; \\ 0, & \text { otherwise. }\end{cases}
$$

(The symbol rng denotes the range of operators and $A^{-1 / 2}$ denotes the inverse of $A^{1 / 2}$ on its range.)

Lemma 1. Let $T \in B(H)^{+}$be arbitrary and $P$ be a rank-one projection on $H$. We have $T \# P=\sqrt{\lambda(T, P)} P$

In what follows, for arbitrary vectors $x, y \in H$ we shall use the notation $x \otimes y$ for the operator defined by

$$
(x \otimes y) z=\langle z, y\rangle x \quad(z \in H) .
$$

Proof. First let $T \in B(H)^{+}$be invertible. Pick a unit vector $x$ from the range of $P$ so that $P=x \otimes x$. Using (v) and the formula (3) one can easily verify that

$$
T \# P=\frac{1}{\left\|T^{-1 / 2} x\right\|} x \otimes x=\sqrt{\lambda(T, P)} P .
$$

In particular, we obtain that for invertible $T$ the geometric mean $T \# P$ is a scalar multiple of $P$. If $T \in B(H)^{+}$is arbitrary, then applying (iii) we deduce that $T \# P$ is again a scalar multiple of $P$. It then follows from the original definition (11) that we have

$$
T \# P=\max \left\{\delta P: \delta \in \mathbb{R}_{+},\left[\begin{array}{cc}
T & \delta P \\
\delta P & P
\end{array}\right] \geq 0\right\} .
$$

Clearly, the inequality

$$
\left\langle\left[\begin{array}{cc}
T & \delta P \\
\delta P & P
\end{array}\right]\left[\begin{array}{l}
u \\
v
\end{array}\right],\left[\begin{array}{l}
u \\
v
\end{array}\right]\right\rangle \geq 0
$$

is equivalent to

$$
\langle T u, u\rangle+\delta\langle P v, u\rangle+\delta\langle P u, v\rangle+\langle P v, v\rangle \geq 0
$$

for all $u, v \in H$. This can be rewritten as

$$
\langle T u, u\rangle+\|P(v+\delta u)\|^{2}-\delta^{2}\|P u\|^{2} \geq 0,
$$

which holds for all $u, v \in H$ if and only if

$$
\langle T u, u\rangle-\delta^{2}\|P u\|^{2} \geq 0,
$$

or equivalently, $\delta^{2} P \leq T$. By (2) it follows that $T \# P=\sqrt{\lambda(T, P)} P$ as we have asserted.

In the proof of the theorem we shall also use the following lemma.

Lemma 2. Let $B \in B(H)^{+}$be invertible. If $Q$ is a nonzero projection and $\lambda$ is a positive real number such that $Q B Q=\lambda Q$ and $Q B^{-1} Q=(1 / \lambda) Q$, then $\operatorname{rng} Q$ is a subspace of the eigensubspace of $B$ corresponding to the eigenvalue $\lambda$. 
Proof. We can suppose without any loss of generality that $\lambda=1$. Let $x$ be any unit vector from the range of $Q$. Multiplying the equations $Q B Q=Q$ and $Q B^{-1} Q=Q$ by $P_{x}=x \otimes x$ from the left and also from the right we obtain

$$
x \otimes x \cdot B \cdot x \otimes x=x \otimes x, \quad x \otimes x \cdot B^{-1} \cdot x \otimes x=x \otimes x .
$$

These imply $\langle B x, x\rangle=1$ and $\left\langle B^{-1} x, x\right\rangle=1$. Consider the Cauchy-Schwarz inequality for the new inner product $(u, v)=\left\langle B^{-1} u, v\right\rangle(u, v \in H)$. Insert $u=B x$ and $v=x$. As $x$ is a unit vector, we see that there is equality in the corresponding inequality

$$
\left|\left\langle B^{-1} B x, x\right\rangle\right|^{2} \leq\left\langle B^{-1} B x, B x\right\rangle\left\langle B^{-1} x, x\right\rangle .
$$

This gives us that $B x$ is a nonzero scalar multiple of $x$. It is clear that this scalar is necessarily 1 . So, we have $B x=x$ for any unit vector $x$ from the range of $Q$. This proves the assertion.

We are now in a position to prove the theorem.

Proof of theorem. In the first step we show that $\phi$ preserves the invertible elements of $B(H)^{+}$in both directions; i.e., for any $A \in B(H)^{+}$we have

$$
A \text { is invertible } \Longleftrightarrow \phi(A) \text { is invertible. }
$$

To show this, it is clearly enough to verify the following characterization of the invertible elements: the operator $A \in B(H)^{+}$is invertible if and only if $A \# B(H)^{+}=$ $\left\{A \# B: B \in B(H)^{+}\right\}=B(H)^{+}$. By (v), the sufficiency is trivial. Now let $A$ be noninvertible. Applying (v) again, we see that for any invertible $B \in B(H)^{+}$, the operator $A \# B=B \# A$ is noninvertible. Next, using (i), one can deduce that $A \# B$ is noninvertible for every $B \in B(H)^{+}$. This proves the asserted characterization of invertibility and hence that $\phi$ preserves the invertible elements in both directions. In particular, we have that $\phi(I)$ is an invertible positive operator. Considering the map

$$
A \longmapsto \phi(I)^{-1 / 2} \phi(A) \phi(I)^{-1 / 2}
$$

we have a bijective transformation of $B(H)^{+}$which, as a consequence of the transfer property (ii), preserves the geometric mean and has the additional property that it sends $I$ to $I$. Without loss of generality, in what follows we assume that $\phi$ already has this property.

We next assert that

$$
\phi\left(T^{2}\right)=\phi(T)^{2}
$$

holds for every $T \in B(H)^{+}$. Indeed, we compute

$$
\phi(T)^{1 / 2}=I \# \phi(T)=\phi(I) \# \phi(T)=\phi(I \# T)=\phi\left(T^{1 / 2}\right)
$$

from which (4) follows. In particular, we obtain that $\phi$ preserves the projections in both directions; i.e., for any $A \in B(H)^{+}$we have

$$
A \text { is a projection } \Longleftrightarrow \phi(A) \text { is a projection. }
$$

From Proposition 2 in 4 we learn that for any two projections $P, Q$ on $H$ we have $P \# Q=P \wedge Q$. Hence, we can infer that $\phi$ when restricted onto the set of all projections on $H$ gives a lattice automorphism.

Pick an invertible $A \in B(H)^{+}$. By (v) we have $A \# A^{-1}=I$. This gives us that $\phi(A) \# \phi\left(A^{-1}\right)=I$. Applying (v) again, one can easily verify that

$$
\phi\left(A^{-1}\right)=\phi(A)^{-1} .
$$


We now show that

$$
\phi(T A T)=\phi(T) \phi(A) \phi(T)
$$

holds for an arbitrary $T \in B(H)^{+}$and invertible $A \in B(H)^{+}$. Let $B=$ $A^{-1 / 2}\left(A^{1 / 2} T A^{1 / 2}\right)^{2} A^{-1 / 2}$. Easy computation shows that $B=T A T$ and $T=$ $A^{-1} \# B$. Therefore, we have

$$
\phi(T)=\phi\left(A^{-1} \# B\right)=\phi(A)^{-1} \# \phi(B),
$$

which gives us that

$$
\phi(T)=\phi(A)^{-1 / 2}\left(\phi(A)^{1 / 2} \phi(B) \phi(A)^{1 / 2}\right)^{1 / 2} \phi(A)^{-1 / 2} .
$$

Multiplying by $\phi(A)^{1 / 2}$ from the left and from the right and taking the square, we get

$$
\phi(A)^{1 / 2} \phi(T) \phi(A) \phi(T) \phi(A)^{1 / 2}=\phi(A)^{1 / 2} \phi(B) \phi(A)^{1 / 2} .
$$

This implies

$$
\phi(T) \phi(A) \phi(T)=\phi(B)=\phi(T A T)
$$

as we have asserted.

Introduce an operation $\circ$ on $B(H)^{+}$by the formula $A \circ B=A^{1 / 2} B A^{1 / 2}$. Concerning Hilbert space effects, this operation is called the sequential product, and it was defined by Gudder and Nagy in [5. Applying (6) and (5) we obtain the equality

$$
\phi(T \circ A)=\phi(T) \circ \phi(A)
$$

for an arbitrary $T \in B(H)^{+}$and invertible $A \in B(H)^{+}$. The operation $\circ$ has many interesting properties. For example, it follows from [5, Corollary 3] that commutativity with respect to the sequential product is equivalent to the commutativity with respect to the usual operator product. Therefore, we infer that $\phi$ preserves the commutativity of the invertible elements in both directions. This means that for any invertible $A, B \in B(H)^{+}$we have

$$
A B=B A \Longleftrightarrow \phi(A) \phi(B)=\phi(B) \phi(A) .
$$

As a consequence, we obtain that $\phi$ maps nonzero scalar operators to nonzero scalar operators. We show that $\phi(0)=0$. In fact, using the formula (v) one can easily check that the zero operator can be characterized, for example, in the following way: for $B \in B(H)^{+}$we have $B=0$ if and only if $A \# B=B$ holds for every invertible $A \in B(H)^{+}$. By this characterization we have $\phi(0)=0$. It follows from the above properties of $\phi$ that there is a bijective function $f: \mathbb{R}_{+} \rightarrow \mathbb{R}_{+}$with $f(0)=0$ such that

$$
\phi(\lambda I)=f(\lambda) I
$$

holds for every $\lambda \in \mathbb{R}_{+}$. One can easily verify that $f$ is multiplicative. In fact, this is a straightforward consequence of the equation $f(\lambda) f(\mu) f(\lambda)=f(\lambda \mu \lambda)$ which follows from (7).

In the next part of the proof we show that $\phi$ preserves the orthogonality between projections. Let $\lambda \neq \mu$ be positive real numbers. Pick mutually orthogonal nonzero projections $P, Q$ on $H$ whose sum is $I$. Set $A=\lambda P+\mu Q$. Consider the projections $P^{\prime}=\phi(P)$ and $Q^{\prime}=\phi(Q)$. As $A$ is invertible, we can compute

$$
\begin{gathered}
P^{\prime} \phi(A) P^{\prime}=\phi(P) \phi(A) \phi(P)=\phi(P A P) \\
=\phi(\lambda P)=\phi(P(\lambda I) P)=P^{\prime} \phi(\lambda I) P^{\prime}=f(\lambda) P^{\prime}
\end{gathered}
$$


and, similarly,

$$
P^{\prime} \phi(A)^{-1} P^{\prime}=\phi\left(P A^{-1} P\right)=\phi(P((1 / \lambda) I) P)=(1 / f(\lambda)) P^{\prime} .
$$

By Lemma 2 we obtain that the range of $P^{\prime}$ is a subspace of the eigensubspace of $\phi(A)$ corresponding to the eigenvalue $f(\lambda)$. Similarly, we get that the range of $Q^{\prime}$ is a subspace of the eigensubspace of $\phi(A)$ corresponding to the eigenvalue $f(\mu)$. Since $f(\lambda) \neq f(\mu)$, it follows that $P^{\prime}$ and $Q^{\prime}$ are mutually orthogonal projections. We already know that $\phi$ when restricted onto the lattice of projections is a lattice automorphism. Therefore, we have

$$
P^{\prime} \vee Q^{\prime}=\phi(P) \vee \phi(Q)=\phi(P \vee Q)=\phi(I)=I .
$$

These imply that

$$
\phi(\lambda P+\mu Q)=f(\lambda) \phi(P)+f(\mu) \phi(Q),
$$

which holds also when $\lambda=\mu$. We shall use this equation to verify that the function $f$ is the identity on $\mathbb{R}_{+}$. Pick any unit vector $x \in H$. Applying Lemma $\mathbb{1}$, on the one hand we have

$$
A \# P_{x}=\sqrt{\lambda\left(A, P_{x}\right)} P_{x}=\sqrt{\frac{1}{\frac{\|P x\|^{2}}{\lambda}+\frac{\|Q x\|^{2}}{\mu}}} P_{x},
$$

which implies that

$$
\phi(A) \# \phi\left(P_{x}\right)=f\left(\sqrt{\frac{1}{\frac{\|P x\|^{2}}{\lambda}+\frac{\|Q x\|^{2}}{\mu}}}\right) \phi\left(P_{x}\right) .
$$

On the other hand, we have $\phi(A)=f(\lambda) P^{\prime}+f(\mu) Q^{\prime}$. Moreover, $\phi\left(P_{x}\right)$ is a rankone projection and hence there is a unit vector $y \in H$ such that $\phi\left(P_{x}\right)=P_{y}$. So, we can compute

$$
\phi(A) \# \phi\left(P_{x}\right)=\left(f(\lambda) P^{\prime}+f(\mu) Q^{\prime}\right) \# P_{y}=\sqrt{\frac{1}{\frac{\left\|P^{\prime} y\right\|^{2}}{f(\lambda)}+\frac{\left\|Q^{\prime} y\right\|^{2}}{f(\mu)}}} P_{y} .
$$

By the last two displayed equations we get

$$
f\left(\sqrt{\frac{1}{\frac{\|P x\|^{2}}{\lambda}+\frac{\|Q x\|^{2}}{\mu}}}\right)=\sqrt{\frac{1}{\frac{\left\|P^{\prime} y\right\|^{2}}{f(\lambda)}+\frac{\left\|Q^{\prime} y\right\|^{2}}{f(\mu)}}} .
$$

Since $f$ is multiplicative, from this we have immediately that

$$
f\left(\mu\|P x\|^{2}+\lambda\|Q x\|^{2}\right)=f(\mu)\left\|P^{\prime} y\right\|^{2}+f(\lambda)\left\|Q^{\prime} y\right\|^{2} .
$$

This equality holds for all positive real numbers $\lambda, \mu$. Moreover, the only constraint concerning the numbers $\|P x\|^{2}$ and $\|Q x\|^{2}$ is that they are nonnegative with sum 1 (a similar observation holds for $\left\|P^{\prime} y\right\|^{2}$ and $\left\|Q^{\prime} y\right\|^{2}$ as well). Therefore, we can deduce from (8) that the image of any convex combination of two arbitrary positive real numbers $\lambda, \mu$ under $f$ is a convex combination of $f(\lambda)$ and $f(\mu)$. This easily implies that $f$ is a strictly monotone bijection on the set of all positive real numbers and hence it is continuous there. We now recall the discussion in the remark. It follows from the argument described there that $f$ is of the form $f(\lambda)=e^{a(\log \lambda)}$ $(\lambda>0)$, where $a$ is a bijective additive function on $\mathbb{R}$. But, because of the continuity of $f$ on the positive real numbers, the additive function $a$ must also be continuous and hence it is necessarily a linear function. We conclude that $f(\lambda)=\lambda^{c}(0<$ $\lambda$ ) holds for some nonzero constant $c$. Since the coefficients $\|P x\|^{2},\|Q x\|^{2}$ and $\left\|P^{\prime} y\right\|^{2},\left\|Q^{\prime} y\right\|^{2}$ in (8) do not depend on the variables $\lambda$ and $\mu$, we easily obtain that 
$c=1$. This gives us that $f$ is the identity on $\mathbb{R}_{+}$. It then follows from (8) that $\|P x\|^{2}=\left\|P^{\prime} y\right\|^{2}$, which can be rewritten as

$$
\operatorname{tr} P P_{x}=\operatorname{tr} \phi(P) \phi\left(P_{x}\right) .
$$

Here $P$ is an arbitrary nonzero projection and $P_{x}$ is any rank-one projection on $H$. We obtain that $\phi$ when restricted onto the set of all rank-one projections gives a bijection with the property that

$$
\operatorname{tr} P Q=\operatorname{tr} \phi(P) \phi(Q)
$$

holds for any rank-one projections $P, Q$ on $H$. Such transformations are called symmetry transformations in quantum mechanics. According to a celebrated theorem of Wigner, we obtain that there exists a unitary or antiunitary operator $U$ on $H$ such that

$$
\phi(P)=U P U^{*}
$$

holds for every rank-one projection $P$ on $H$. (For generalizations of Wigner's theorem concerning different structures we refer to the Sections 2.1-2.3 of our recent work [6]; see also the references therein.) Considering the transformation

$$
A \longmapsto U^{*} \phi(A) U \quad\left(A \in B(H)^{+}\right),
$$

we can now assume that $\phi$ is the identity on the rank-one projections. It remains to prove that $\phi$ is the identity on the whole set $B(H)^{+}$. To see this, let $A \in B(H)^{+}$ be arbitrary. Pick any rank-one projection $P$ on $H$. We compute

$$
\begin{gathered}
\sqrt{\lambda(\phi(A), P)} P=\phi(A) \# P=\phi(A) \# \phi(P) \\
=\phi(A \# P)=\phi(\sqrt{\lambda(A, P)} P)=f(\sqrt{\lambda(A, P)}) \phi(P)=\sqrt{\lambda(A, P)} P .
\end{gathered}
$$

This implies that $\lambda(\phi(A), P)=\lambda(A, P)$ holds for every rank-one projection $P$ on $H$. Since every positive operator is uniquely determined by its strength function (see [3, Corollary 1]), we obtain $\phi(A)=A$.

To complete the proof observe that we have shown above that the transformation

$$
A \longmapsto\left(U^{*} \phi(I)^{-1 / 2}\right) \phi(A)\left(\phi(I)^{-1 / 2} U\right)
$$

is the identity on $B(H)^{+}$. This clearly proves the statement of the theorem.

\section{REFERENCES}

1. T. Ando, Topics on operator inequalities, Division of Applied Math., Research Institute of Applied Electricity, Hokkaido University, Sapporo, 1978. MR0482378 (58:2451)

2. I. Bengtsson and K. Życzkowski, Geometry of Quantum States. An Introduction to Quantum Entanglement, Cambridge University Press, 2006. MR2230995 (2007k:81001)

3. P. Busch and S.P. Gudder, Effects as functions on projective Hilbert space, Lett. Math. Phys. 47 (1999), 329-337. MR1693743 (2000e:81014)

4. J.-I. Fujii, Arithmetico-geometric mean of operators, Math. Japon. 23 (1978/79), 667-669. MR.529901 (80g:47006)

5. S. Gudder and G. Nagy, Sequentially independent effects, Proc. Amer. Math. Soc. 130 (2002), 1125-1130. MR1873787 (2002i:81014) 
6. L. Molnár, Selected Preserver Problems on Algebraic Structures of Linear Operators and on Function Spaces, Lecture Notes in Mathematics, Vol. 1895, Springer, 2006. MR2267033 (2007g:47056)

7. P. Semrl, Isomorphisms of standard operator algebras, Proc. Amer. Math. Soc. 123 (1995), 1851-1855. MR1242104 (95g:47066)

Institute of Mathematics, University of Debrecen, P.O. Box 12, H-4010 Debrecen, HUNGARY

E-mail address: molnarl@math.klte.hu

URL: http://www.math.klte.hu/ molnarl/ 